\title{
Subsumption Architecture Applied to Flight Control using Composite Rotations
}

\author{
E. Oland, T. S. Andersen, R. Kristiansen \\ UiT - The Arctic University of Norway, Faculty of Engineering Science and Technology, Norway
}

\begin{abstract}
In this paper the subsumption theory is applied to flight control through composite rotations where multiple tasks can be defined as simple rotations. The tasks can then be arranged as a hierarchy, where the primary task is always fully pursued, and conflicting lower level tasks are removed by the primary rotation. The concept is applied to a group of UAVs that move through an urban terrain while avoiding collisions with the ground, the buildings and other UAVs as they track a desired waypoint.
\end{abstract}

Key words: Guidance, navigation and control of vehicles, autonomous systems, application of nonlinear analysis and design.

\section{Introduction}

To facilitate the design of a truly autonomous agent, the controller must enable the agent to perform multiple tasks in a complex dynamic environment. The layered approach to behavioral control is presented in [3], where multiple tasks are arranged in a hierarchy and where a higher level task subsumes (or suppresses) lower level tasks. This ensures that the primary task is always fully pursued, while the lower level tasks are fully pursued as the higher level tasks are completed. This layered structure enables the complex problem of behavioral control to be defined as several simple tasks that are combined using subsumption. By adding an addition layer (or task), the competence of the agent is further augmented, thus increasing the apparent level of intelligence. The subsumption architecture has later resulted in the Null-Space-based Behavioral (NSB) control method as presented in [1]. It adheres to the principle of layered control and define each task as a desired velocity vector. The tasks (or velocity vectors) are then arranged in a hierarchy, where conflicting lower level velocity components are projected onto the nullspace, thus removing their contribution from the resulting reference velocity vector. For fixed-wing Unmanned Aerial Vehicles (UAVs), the use of a reference velocity vector is not directly applicable since a fixed-wing UAV is underactu-

\footnotetext{
* This paper was not presented at any IFAC meeting. Corresponding author E. Oland. Tel. +47-97725709.

Email addresses: espen.oland@uit.no (E. Oland), tom.s.andersen@uit.no (T. S. Andersen), raymond.kristiansen@uit.no (R. Kristiansen).
}

ated with only translational control in one direction. Instead of defining the tasks as velocity vectors, it is possible to design them directly as rotations that are multiplied together, producing a desired orientation that ensures that the primary task is always fully pursued.

The main idea and contribution of this paper is that a rotation between two frames can be decomposed into a composite rotation where each rotation can be treated as a task according to the subsumption architecture. Consider the composite quaternion rotation

$$
\mathbf{q}_{D, A}=\mathbf{q}_{D, C} \otimes \mathbf{q}_{C, B} \otimes \mathbf{q}_{B, A}
$$

which performs a rotation from frame $A$ to frame $D$ through the intermediate frames $B$ and $C$. By defining each quaternion relative to the previous quaternion, the last quaternion will always define the direction that a vector rotated by $\mathbf{q}_{D, A}$ is pointing in, independently of the previous rotations. This fact can be used to arrange the tasks into a hierarchy through composite rotations. As the tasks are completed, their quaternion is set to the identity quaternion such that their contribution to the resulting quaternion is suppressed, enabling a fusion of tasks that facilitates behavioral control. Each task is in this paper designed by mapping position vectors describing the task (e.g. error between current and desired position) from $\mathbb{R}^{3}$ to $\mathbb{R} \times \mathrm{S}^{3}$, providing the quaternion that describes the direction that the velocity vector must be pointed in to solve the given task.

This paper applies the subsumption architecture to perform collision (and ground) avoidance while tracking a 
desired waypoint. The problem of collision avoidance has received much attention the last decades. The most well known approach is the use of potential fields as presented in [5]. Even though the potential field approach is intuitively simple and appealing, it suffers from several problems such as local minima, inability to pass through narrow gaps, and the possibility of becoming trapped [6]. These facts motivated further research and resulted in the navigation function [10] which addresses the local minima problem, and has later has been extended for nonholonomic vehicles in [13] and applied for nonholonomic aircraft-like vehicles in [11]. The problems that were pointed out in [6] is applicable to many of the local collision avoidance methods. As such, they deserve special attention. In this paper, a detection box is applied for obstacle detection, similarly to the vector histogram as proposed in [2], and enables an agent to pass through narrow gaps. Furthermore, the subsumption architecture facilitates an arbitrary number of layers, and as such, even though it is not implemented in this paper, it is possible to add an addition layer that serves as a global navigation function that defines no-fly-zones wherever an agent can become trapped, i.e. the framework allows for an avoidance of local minima.

\section{Modeling}

The time derivative of a vector is given as $\dot{\mathbf{x}}=d \mathbf{x} / d t$, the Euclidean length is written as $\|\mathbf{x}\|=\sqrt{\mathbf{x}^{\top} \mathbf{x}}$ and $\dagger$ denotes the Moore-Penrose pseudoinverse. Superscript denotes the reference frame of a vector where $b$ denotes the body frame, $n$ denotes the North East Down (NED) frame, $w$ denotes the wind frame, $d$ denotes the desired frame, $e$ denotes the position error frame, $c$ denotes the collision avoidance frame and $h$ denotes the ground avoidance frame. The rotation matrix is denoted $\mathbf{R}_{A}^{B} \in \mathrm{SO}(3)=\left\{\mathbf{R} \in \mathbb{R}^{3 \times 3}: \mathbf{R}^{\top} \mathbf{R}=\mathbf{I}, \operatorname{det}(\mathbf{R})=\right.$ $1\}$, which rotates a vector from frame $A$ to frame $B$ and where $\mathbf{I}$ denotes the identity matrix. The angular velocity vector is denoted $\boldsymbol{\omega}_{A, B}^{C}$, which represents the angular velocity of frame $B$ relative to frame $A$ referenced in frame $C$. Angular velocities between different frames can be added together as $\boldsymbol{\omega}_{A, D}^{C}=\boldsymbol{\omega}_{A, B}^{C}+\boldsymbol{\omega}_{B, D}^{C}$. The time derivative of the rotation matrix is found as $\dot{\mathbf{R}}_{A}^{C}=\mathbf{R}_{A}^{C} \mathbf{S}\left(\boldsymbol{\omega}_{C, A}^{A}\right)$ where the cross product operator $\mathbf{S}(\cdot)$ is such that for two arbitrarily vectors $\mathbf{v}_{1}, \mathbf{v}_{2} \in \mathbb{R}^{3}$, $\mathbf{S}\left(\mathbf{v}_{1}\right) \mathbf{v}_{2}=\mathbf{v}_{1} \times \mathbf{v}_{2}$. The rotation matrix can be parameterized using quaternions, where the quaternion that rotates from frame $A$ to frame $C$ is denoted $\mathbf{q}_{C, A} \in$ $\mathrm{S}^{3}=\left\{\mathbf{q} \in \mathbb{R}^{4}: \mathbf{q}^{\top} \mathbf{q}=1\right\}$ and can be written as $\mathbf{q}_{C, A}=\left[\eta_{C, A} \boldsymbol{\epsilon}_{C, A}^{\top}\right]^{\top}=\left[\cos \left(\frac{\vartheta_{C, A}}{2}\right) \mathbf{k}_{C, A}^{\top} \sin \left(\frac{\vartheta_{C, A}}{2}\right)\right]^{\top}$ where $\eta_{C, A}$ is the scalar part and $\boldsymbol{\epsilon}_{C, A} \in \mathbb{R}^{3}$ is the vector part enabling the the rotation matrix to be constructed as $\mathbf{R}_{A}^{C}=\mathbf{I}+2 \eta_{C, A} \mathbf{S}\left(\boldsymbol{\epsilon}_{C, A}\right)+2 \mathbf{S}^{2}\left(\boldsymbol{\epsilon}_{C, A}\right)$. The quaternion performs a rotation of an angle $\vartheta_{C, A}$ around the unit vector $\mathbf{k}_{C, A}$ and the inverse quaternion is defined as $\mathbf{q}_{A, C}=$
$\left[\eta_{C, A}-\boldsymbol{\epsilon}_{C, A}^{\top}\right]^{\top}$. Composite rotations are found using the quaternion product as $\mathbf{q}_{C, E}=\mathbf{q}_{C, A} \otimes \mathbf{q}_{A, E}$ which ensures that the resulting quaternion maintains the unit length property. The translational kinematics can be defined as ( $c f$. [12]) $\dot{\mathbf{p}}^{n}=\mathbf{R}_{b}^{n} \mathbf{v}^{b}$ and $\mathbf{v}_{r}^{b}=\mathbf{v}^{b}-\mathbf{R}_{n}^{b} \mathbf{w}^{n}$ where $\mathbf{p}^{n}$ is the position vector, $\mathbf{v}^{b}$ is the velocity vector relative to the ground, $\mathbf{v}_{r}^{b}$ is the velocity vector relative to the surrounding air and $\mathbf{w}^{n}$ is the velocity of the wind. The relative velocity vector $\left(\mathbf{v}_{r}^{b}\right)$ can be rotated to the wind frame as $\mathbf{v}_{r}^{w}=\mathbf{R}_{b}^{w} \mathbf{v}_{r}^{b}=\left[\begin{array}{lll}V_{a} & 0 & 0\end{array}\right]^{\top}$ where $V_{a}$ is the airspeed. By pointing the airspeed in a desired direction and move with a desired airspeed, any control objective can be fulfilled. Hence, a quaternion error function between the wind frame and the desired frame can be defined as $(c f .[7]) \mathbf{e}_{q \pm}=\left[1 \mp \eta_{d, w} \boldsymbol{\epsilon}_{d, w}\right] \in \mathrm{S}_{e}^{3}$, where $\mathrm{S}_{e}^{3}=\left\{\left[1 \mp \eta_{d, w} \boldsymbol{\epsilon}_{d, w}^{\top}\right]^{\top} \mid \mathbf{q}_{d, w} \in \mathrm{S}^{3}\right\}$, and a speed error can be defined as $\tilde{V}=V_{a}-V_{d}$ where $V_{d}$ is a desired airspeed. The control objective can now be formalized as that of making $\left(\tilde{V}, \mathbf{e}_{q \pm}, \boldsymbol{\omega}_{d, w}^{b}\right) \rightarrow(0, \mathbf{0}, \mathbf{0})$ which can be fulfilled by using the results from [8]. This paper is concerned with the design of the desired quaternion, angular velocity and acceleration $\left(\mathbf{q}_{n, d}, \boldsymbol{\omega}_{n, d}^{d}, \dot{\boldsymbol{\omega}}_{n, d}^{d}\right)$ using the subsumption architecture.

\section{Main Results}

This paper considers three tasks that can be arranged into a hierarchy and represented using quaternions as:

- Task 1: Ground avoidance, $\mathbf{q}_{c, h}$

- Task 2: Collision avoidance, $\mathbf{q}_{e, c}$

- Task 3: Waypoint tracking, $\mathbf{q}_{n, e}$;

and can be combined into a quaternion and corresponding angular velocity as

$$
\begin{aligned}
\mathbf{q}_{n, h} & =\mathbf{q}_{n, e} \otimes \mathbf{q}_{e, c} \otimes \mathbf{q}_{c, h} \\
\boldsymbol{\omega}_{n, h}^{h} & =\mathbf{R}_{e}^{h} \boldsymbol{\omega}_{n, e}^{e}+\mathbf{R}_{c}^{h} \boldsymbol{\omega}_{e, c}^{c}+\boldsymbol{\omega}_{c, h}^{h} .
\end{aligned}
$$

The primary task is ground avoidance which shall ensure that the UAV never collides with the ground. The secondary task is collision avoidance which shall ensure that the UAV does not collide with buildings nor other $\mathrm{UAVs}^{1}$. The tertiary task is waypoint tracking, where the UAV shall reach a desired waypoint. All tasks are defined using position vectors in the NED frame. As a task is completed, its quaternion is set equal to the identity quaternion, while its angular velocity is set to zero. This enables multiple tasks to be fused together producing a quaternion and angular velocity that facilitates behavioral control. With basis in Figure 1, let the position er-

\footnotetext{
1 Note that this paper only proves collision avoidance for static obstacles. In the simulation, each UAV is encompassed with a protective sphere to account for this.
} 


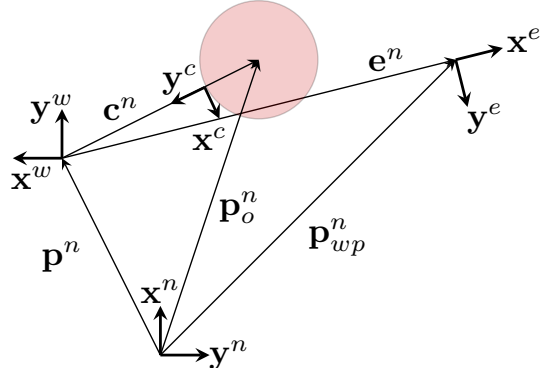

Fig. 1. Position vectors relative to the NED frame. In the case of waypoint tracking, the objective is to align the wind frame with the position error frame which will make the position error go towards zero. In the case of collision avoidance, the objective is to align the wind frame with the collision avoidance frame, resulting in a circular motion around obstacles. By combining the two quaternions, it is possible to move to a desired waypoint without collision.

ror frame be defined through the relation

$$
\mathbf{e}^{e}:=\left[\begin{array}{lll}
\left\|\mathbf{e}^{n}\right\| & 0 & 0
\end{array}\right]^{\top}=\mathbf{R}_{n}^{e} \mathbf{e}^{n}=\mathbf{R}_{n}^{e}\left(\mathbf{p}_{w p}^{n}-\mathbf{p}^{n}\right)
$$

where the objective is to make $\mathbf{e}^{e} \rightarrow \mathbf{0}$ and switch to a new waypoint before the origin is reached. To facilitate waypoint tracking, consider the two assumptions:

Assumption $1 V_{a} \geq \beta_{v}>0$.

Assumption $2 \mathrm{w}^{n}=\mathbf{0}$.

Assumption 1 states that the UAV has a positive speed above stall speed which is a requirement for flight, something that can be enforced through a speed controller. Assumption 2 simplifies the waypoint problem and will later be removed by the quaternion $\left(\mathbf{q}_{h, d}\right)$ that compensates for the wind.

Lemma 1 Let Assumptions 1 and 2, $\left\|\mathbf{e}^{n}\left(t_{0}\right)\right\|>\delta>0$ hold, and let a set be defined as $\mathcal{H}(\delta, \Delta):=\left\{\mathbf{x} \in \mathbb{R}^{3} \mid \delta \leq\right.$ $\left.\left\|\mathbf{e}^{n}\right\| \leq \Delta\right\}$ where $\delta>0$ represents an inner radius and $\Delta>\delta>0$ an outer radius. Given the position error as in (4) and by tracking the quaternion and angular velocity

$$
\begin{aligned}
& \mathbf{q}_{n, e}=\left[\cos \left(\frac{\vartheta_{n, e}}{2}\right) \mathbf{k}_{n, e}^{\top} \sin \left(\frac{\vartheta_{n, e}}{2}\right)\right]^{\top} \\
& \vartheta_{n, e}=\cos ^{-1}\left(\frac{\mathbf{e}^{e} \cdot \mathbf{e}^{n}}{\left\|\mathbf{e}^{n}\right\|^{2}}\right) \quad \mathbf{k}_{n, e}=\frac{\mathbf{e}^{e} \times \mathbf{e}^{n}}{\left\|\mathbf{e}^{e} \times \mathbf{e}^{n}\right\|} \\
& \boldsymbol{\omega}_{n, e}^{e}=\mathbf{S}^{\dagger}\left(\mathbf{e}^{e}\right) \mathbf{R}_{b}^{e} \mathbf{v}^{b}
\end{aligned}
$$

then the set $\mathcal{H}(\delta, \Delta)$ is uniformly asymptotically stable.

Proof 1 The proof is given in Appendix A.

To facilitate collision avoidance, consider Figure 1. By moving perpendicular to the line of sight vector to the closest obstacle $\left(\mathbf{c}^{n}\right)$, collisions can be avoided. By choosing the sign of the collision avoidance frame, the UAV can move either to the left or the right around an obstacle. This paper applies a detection box that is fixed to the wind frame of the UAV and contains two sectors. If an obstacle is detected in the left sector, the UAV moves to the right, and left otherwise. The collision avoidance frame be defined through the relation $\mathbf{c}^{c}=\left[0 \pm\left\|\mathbf{c}^{e}\right\| 0\right]^{\top}=\mathbf{R}_{e}^{c} \mathbf{R}_{n}^{e} \mathbf{c}^{n}$ where $\mathbf{R}_{e}^{c}$ is designed using quaternions. Note that the vector $\mathbf{c}^{n}$ is first rotated to the position error frame before designing the collision avoidance frame. This dependence is what enables the application of subsumption theory through composite rotations. For ground avoidance, the line of sight vector to the ground is first rotated to the collision avoidance frame before designing the ground avoidance frame.

Lemma 2 Let Assumptions 1 and 2 hold. Given the position error between the closest obstacle $\left(\mathbf{p}_{o}^{n}\right)$ and the $\mathrm{UAV}$ as $\mathbf{c}^{e}=\mathbf{R}_{n}^{e} \mathbf{c}^{n}=\mathbf{R}_{n}^{e}\left(\mathbf{p}_{o}^{n}-\mathbf{p}^{n}\right)$, and by following the attitude and angular velocity

$$
\begin{aligned}
\mathbf{q}_{e, c} & =\left[\cos \left(\frac{\vartheta_{e, c}}{2}\right) \mathbf{k}_{e, c}^{\top} \sin \left(\frac{\vartheta_{e, c}}{2}\right)\right]^{\top} \\
\boldsymbol{\omega}_{e, c}^{c} & =\mathbf{S}^{\dagger}\left(\mathbf{c}^{c}\right)\left(\mathbf{R}_{e}^{c} \mathbf{S}\left(\boldsymbol{\omega}_{n, e}^{e}\right) \mathbf{R}_{n}^{e} \mathbf{c}^{n}-\mathbf{R}_{n}^{c} \dot{\mathbf{c}}^{n}\right) \\
\vartheta_{e, c} & =\cos ^{-1}\left(\frac{\mathbf{c}^{c} \cdot \mathbf{c}^{e}}{\left\|\mathbf{c}^{e}\right\|^{2}}\right) \quad \mathbf{k}_{e, c}=\frac{\mathbf{c}^{c} \times \mathbf{c}^{e}}{\left\|\mathbf{c}^{c} \times \mathbf{c}^{e}\right\|} \\
\mathbf{c}^{c} & =\left[0 \pm\left\|\mathbf{c}^{e}\right\| 0\right]^{\top}=\mathbf{R}_{e}^{c} \mathbf{R}_{n}^{e} \mathbf{c}^{n}=\mathbf{R}_{e}^{c} \mathbf{c}^{e}, \\
\text { then }\left\|\mathbf{c}^{c}\right\| & \geq \beta_{c}>0 \forall t \geq t_{0} .
\end{aligned}
$$

Proof 2 The proof is given in Appendix B.

Lemma 3 Let Assumptions 1 and 2 hold. Given the position error between the closest point on the ground $\left(\mathbf{p}_{g}^{n}\right)$ and the $\mathrm{UAV}$ as $\mathbf{h}^{c}=\mathbf{R}_{e}^{c} \mathbf{R}_{n}^{e} \mathbf{h}^{n}=\mathbf{R}_{n}^{c}\left(\mathbf{p}_{g}^{n}-\mathbf{p}^{n}\right)$, and by following the attitude and angular velocity

$$
\begin{aligned}
& \mathbf{q}_{c, h}=\left[\cos \left(\frac{\vartheta_{c, h}}{2}\right) \mathbf{k}_{c, h}^{\top} \sin \left(\frac{\vartheta_{c, h}}{2}\right)\right]^{\top} \\
& \boldsymbol{\omega}_{c, h}^{h}=\mathbf{S}^{\dagger}\left(\mathbf{h}^{h}\right)\left(\mathbf{R}_{c}^{h} \mathbf{S}\left(\boldsymbol{\omega}_{e, c}^{c}\right) \mathbf{R}_{n}^{c} \mathbf{h}^{n}+\mathbf{R}_{e}^{h} \mathbf{S}\left(\boldsymbol{\omega}_{n, e}^{e}\right) \mathbf{R}_{n}^{e} \mathbf{h}^{n}\right. \\
&\left.-\mathbf{R}_{b}^{h} \dot{\mathbf{h}}^{n}\right) \\
& \vartheta_{c, h}=\cos ^{-1}\left(\frac{\mathbf{h}^{h} \cdot \mathbf{h}^{c}}{\left\|\mathbf{h}^{c}\right\|^{2}}\right) \quad \mathbf{k}_{c, h}=\frac{\mathbf{h}^{h} \times \mathbf{h}^{c}}{\left\|\mathbf{h}^{h} \times \mathbf{h}^{c}\right\|} \\
& \mathbf{h}^{h}=\left[\begin{array}{ll}
0 & 0\left\|\mathbf{h}^{c}\right\|
\end{array}\right]^{\top}=\mathbf{R}_{c}^{h} \mathbf{R}_{e}^{c} \mathbf{R}_{n}^{e} \mathbf{h}^{n}=\mathbf{R}_{c}^{h} \mathbf{h}^{c} \\
& \text { then }\left\|\mathbf{h}^{h}\right\| \geq \beta_{h}>0 \forall t \geq t_{0} .
\end{aligned}
$$

Proof 3 The proof follows the same lines as Lemma 2 and is therefore omitted.

Remark 1 The lower bounds $\beta_{c}$ and $\beta_{h}$ are functions of speed, detection range, actuator constraints and the rotational control law. Hence, the detection range must be sufficiently long to account for this to ensure collisionfree trajectories. 
Remark 2 By setting the $z$ component of $\mathbf{c}^{n}$ and the $x$ and $y$ components of $\mathbf{h}^{n}$ to zero before calculating the quaternions, the quaternion $\mathbf{q}_{c, h}$ becomes constrained to the vertical plane and only subsumes the flight path angle. This implies that the vehicle may always avoid simultaneously the ground (controlling the flight path using $\mathbf{q}_{c, h}$ ) and an obstacle defined as a vertical cylinder (by controlling the heading angle using $\left.\mathbf{q}_{e, c}\right)$, or to simultaneously track a ground target while avoiding the ground.

Theorem 1 Let Assumptions 1 and 2 hold. By following the attitude and angular velocity (2)-(3) where the terms are given in lemmas 1, 2 and 3, then $\left\|\mathbf{c}^{c}\right\| \geq \beta_{c}>$ $0,\left\|\mathbf{h}^{h}\right\| \geq \beta_{h}>0 \forall t \geq t_{0}$ and $\left\|\mathbf{e}^{e}\right\| \rightarrow \mathcal{H}(\delta, \Delta)$.

Proof 4 The proof is given in Appendix $C$.

To remove Assumption 2, let a wind compensation quaternion be designed as

$$
\begin{aligned}
& \mathbf{q}_{h, d}=\left[\cos \left(\frac{\vartheta_{h, d}}{2}\right) \mathbf{k}_{h, d}^{\top} \sin \left(\frac{\vartheta_{h, d}}{2}\right)\right]^{\top} \\
& \vartheta_{h, d}=\cos ^{-1}\left(\frac{\mathbf{v}^{b} \cdot \mathbf{v}_{r}^{b}}{\left\|\mathbf{v}^{b}\right\|\left\|\mathbf{v}_{r}^{b}\right\|}\right) \quad \mathbf{k}_{h, d}=\frac{\mathbf{v}^{b} \times \mathbf{v}_{r}^{b}}{\left\|\mathbf{v}^{b} \times \mathbf{v}_{r}^{b}\right\|}
\end{aligned}
$$

which compensates for the wind and where the angular velocity and acceleration are found using linear filters. The tasks from the subsumption architecture defined through (2) and (3) together with wind compensation produce the desired quaternion and angular velocity as

$$
\begin{aligned}
\mathbf{q}_{n, d} & =\mathbf{q}_{n, e} \otimes \mathbf{q}_{e, c} \otimes \mathbf{q}_{c, h} \otimes \mathbf{q}_{h, d} \\
\boldsymbol{\omega}_{n, d}^{d} & =\mathbf{R}_{e}^{d} \boldsymbol{\omega}_{n, e}^{e}+\mathbf{R}_{c}^{d} \boldsymbol{\omega}_{e, c}^{c}+\mathbf{R}_{h}^{d} \boldsymbol{\omega}_{c, h}^{h}+\boldsymbol{\omega}_{h, d}^{d}
\end{aligned}
$$

which also shows the extendability of the proposed approach. The addition of a new task is simply done by expanding the quaternion product, and where the resulting angular velocity is found by adding an additional term.

Remark 3 The proofs of this paper are only valid for one static obstacle, and in practice there are many situations that invalidate the presented approach, e.g. the waypoint is too close to the ground, multiple obstacles form a trap for the UAV, a moving obstacle has higher speed than the $\mathrm{UAV}$, etc.

\section{Simulation}

Simulations have been performed with basis in the nonlinear dynamics and controllers from [8]. Seven UAVs shall move through an urban terrain while avoiding collisions with the buildings, the ground and other UAVs. The main wind vector is set to $\mathbf{w}^{n}=\left[\begin{array}{lll}10 & 0 & 0\end{array}\right]^{\top}$ and wind gusts are simulated using the Dryden wind turbulence model with the default settings as in Simulink. The UAVs shall keep 40 meters above the ground and shall

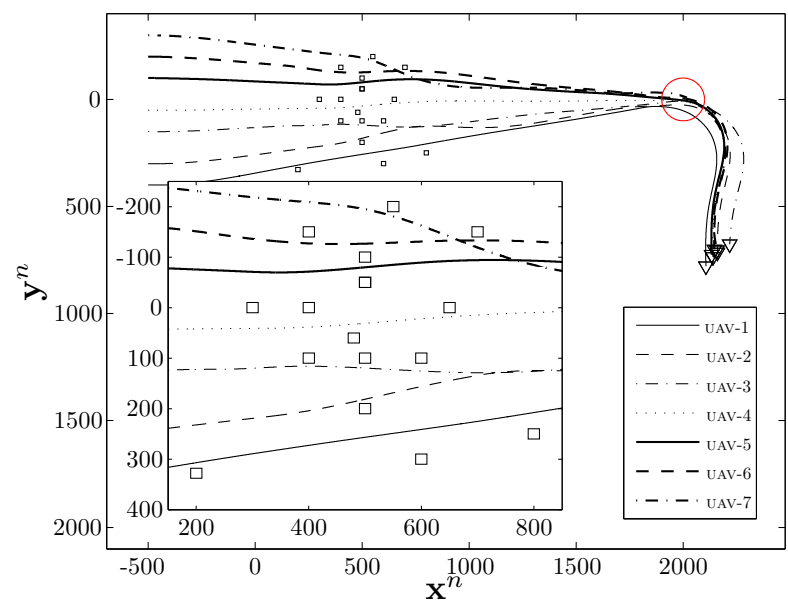

Fig. 2. Seven UAVs move through an urban terrain. The buildings are represented by rectangles, the desired waypoint as a circle and the final positions as triangles.
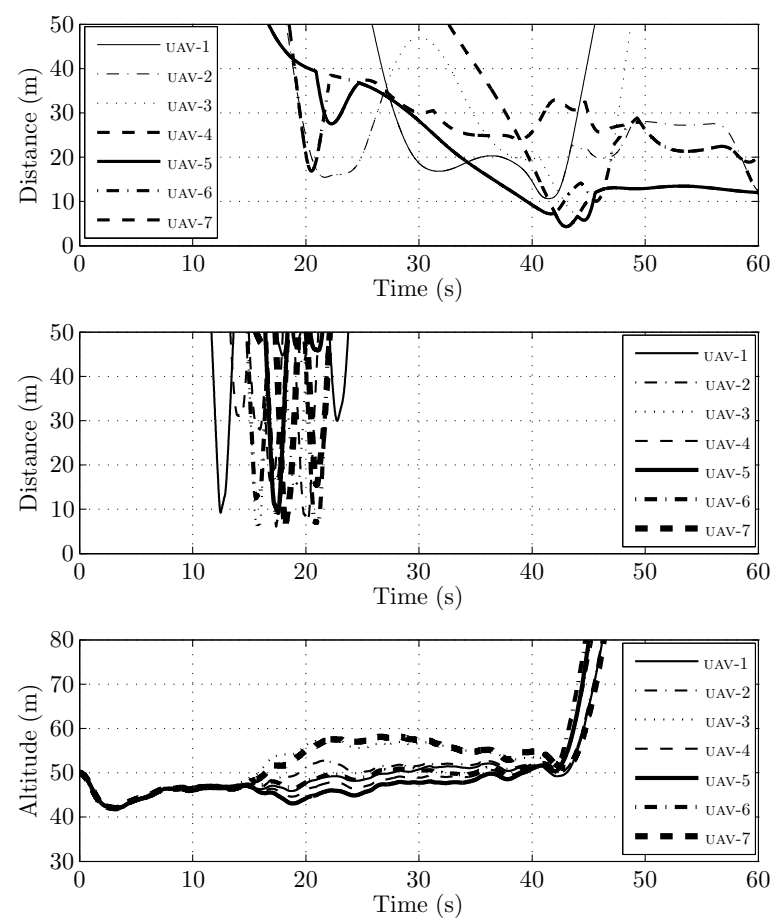

Fig. 3. The top plot shows the shortest distance to the closest UAV, the middle plot shows the shortest distance to the closest obstacle, and the bottom plot shows the altitudes.

keep 5 meters away from the closest obstacle while flying with $V_{d}=50 \mathrm{~m} / \mathrm{s}$. Figure 2 shows the $2 \mathrm{D}$ plot of the simulation, while Figure 3 shows the relative distances. No collisions have occurred and all UAVs reached the waypoint. The altitude of the second waypoint is $1000 \mathrm{~m}$.

\section{Conclusion}

This paper has presented a solution to the problem of maneuvering in a complex dynamical environment us- 
ing the subsumption architecture. Multiple tasks can be defined as simple rotations that are multiplied together producing a desired quaternion. The architecture ensures that the primary task will always be fully pursued while lower level tasks are fully pursued as the higher level tasks are completed.

\section{A Waypoint Tracking}

The following proof first designs the position error frame by taking basis in $\mathbf{e}^{e}=\mathbf{R}_{n}^{e} \mathbf{e}^{n}$, and then the stability of the set $\mathcal{H}(\delta, \Delta)$ is shown by looking at the error function

$$
\mathbf{e}_{\delta}^{e}=\left[\begin{array}{lll}
\left\|\mathbf{e}^{n}\right\|_{\delta} & 0 & 0
\end{array}\right]^{\top}=\mathbf{R}_{n}^{e} \mathbf{e}^{n}
$$

where $\left\|\mathbf{e}^{n}\right\|_{\delta}=\inf _{\mathbf{x} \in \mathcal{H}}\left\|\mathbf{x}-\mathbf{p}^{n}\right\|$ which considers all errors except a small ball around the origin of $\mathbf{e}^{n}=\mathbf{0}$. Consider the error function (4) where the rotation matrix $\mathbf{R}_{n}^{e}$ can be constructed with quaternions using (5). The angular velocity can be found by differentiating (4) resulting in

$$
\dot{\mathbf{e}}^{e}=\mathbf{S}\left(\mathbf{e}^{e}\right) \boldsymbol{\omega}_{n, e}^{e}-\mathbf{R}_{b}^{e} \mathbf{v}^{b}
$$

where $-\mathbf{S}\left(\boldsymbol{\omega}_{n, e}^{e}\right) \mathbf{e}^{e}=\mathbf{S}\left(\mathbf{e}^{e}\right) \boldsymbol{\omega}_{n, e}^{e}, \dot{\mathbf{p}}_{w p}^{n}=\mathbf{0}$ and $\dot{\mathbf{p}}^{n}=$ $\mathbf{R}_{b}^{n} \mathbf{v}^{b}$ have been used. The pseudoinverse of the skew symmetric matrix can be expressed as

$$
\mathbf{S}^{\dagger}\left(\mathbf{e}^{e}\right)=\left[\begin{array}{ccc}
0 & 0 & 0 \\
0 & 0 & -\frac{1}{\left\|\mathbf{e}^{n}\right\|} \\
0 & \frac{1}{\left\|\mathbf{e}^{n}\right\|} & 0
\end{array}\right]
$$

which filters out any components along the $\mathbf{x}^{e}$ axis, such that $\mathbf{S}^{\dagger}\left(\mathbf{e}^{e}\right) \dot{\mathbf{e}}^{e}=\mathbf{0}$. This can be exploited to solve (A.2) for the angular velocity as in (7). Notice that as $\left\|\mathbf{e}^{n}\right\| \rightarrow 0$ the angular velocity $\left\|\boldsymbol{\omega}_{n, e}^{e}\right\| \rightarrow \infty$, such that a switching algorithm must be applied to avoid this. More precisely, as long as $\left\|\mathbf{e}^{n}\right\| \geq \delta>0$, the angular velocity will remain bounded. To that end, the set $\mathcal{H}(\delta, \Delta)$ is defined as a shell around the origin $\mathbf{e}^{n}=\mathbf{0}$, such that the UAV will never reach the interior of the shell which ensures that $\left\|\mathbf{e}^{n}\right\| \geq \delta>0$ making the angular velocity (7) upper bounded as $\left\|\boldsymbol{\omega}_{n, e}^{e}\right\| \leq \frac{1}{\delta} \beta_{V_{\max }}$ where $\beta_{V_{\max }}$ is an upper bound of the velocity vector ${ }^{2}$. Equation (A.1) can be differentiated as $\dot{\mathbf{e}}_{\delta}^{e}=-\mathbf{S}\left(\boldsymbol{\omega}_{n, e}^{e}\right) \mathbf{e}_{\delta}^{e}+$ $\mathbf{R}_{n}^{e}\left(\dot{\mathbf{p}}_{w p}^{n}-\dot{\mathbf{p}}^{n}\right)$ where by using Assumption 2 it follows that $\mathbf{v}^{b}=\mathbf{v}_{r}^{b}=\mathbf{R}_{w}^{b}\left[\begin{array}{lll}V_{a} & 0 & 0\end{array}\right]^{\top}$ with $V_{a} \geq \beta_{v}$ using Assumption 1. The waypoint is a fixed point, such that $\dot{\mathbf{p}}_{w p}^{n}=\mathbf{0}$, enabling the error kinematics to be written

\footnotetext{
${ }^{2}$ It can be shown that $\dot{V}_{a}=-D+\gamma(\cdot)$ where $\gamma(\cdot)$ is a bounded function and $D$ is a positive drag force, such that $\left\|\mathbf{v}_{r}^{w}\right\|=V_{a} \leq \beta_{V_{\max }}$. With $\mathbf{w}^{n}=\mathbf{0},\left\|\mathbf{v}^{b}\right\|=\left\|\mathbf{v}_{r}^{w}\right\| \leq \beta_{V_{\max }}$.
}

as $\dot{\mathbf{e}}_{\delta}^{e}=-\mathbf{S}\left(\boldsymbol{\omega}_{n, e}^{e}\right) \mathbf{e}_{\delta}^{e}-\mathbf{R}_{w}^{e} \mathbf{v}_{r}^{w}$ where the rotation matrix $\mathbf{R}_{w}^{e}=\mathbf{I}+2 \eta_{e, w} \mathbf{S}\left(\boldsymbol{\epsilon}_{e, w}\right)+2 \mathbf{S}^{2}\left(\boldsymbol{\epsilon}_{e, w}\right)$ can be inserted as

$$
\begin{aligned}
\dot{\mathbf{e}}_{\delta}^{e} & =-\mathbf{S}\left(\boldsymbol{\omega}_{n, e}^{e}\right) \mathbf{e}_{\delta}^{e}-\mathbf{v}_{r}^{w} \\
& -\left(2 \eta_{e, w} \mathbf{S}\left(\boldsymbol{\epsilon}_{e, w}\right)+2 \mathbf{S}^{2}\left(\boldsymbol{\epsilon}_{e, w}\right)\right) \mathbf{v}_{r}^{w}
\end{aligned}
$$

where the term $\mathbf{v}_{r}^{w}$ provides damping to the system. Using Assumption 2, it follows that $\mathbf{q}_{n, d}=\mathbf{q}_{n, e}$, i.e. there is no quaternion for wind compensation. Thus, by making

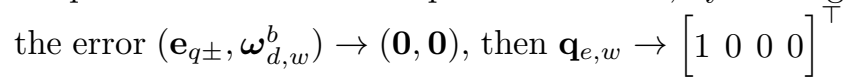
such that the last term in (A.4) will disappear. The system can now be written on cascaded form as

$$
\begin{aligned}
\dot{\mathbf{x}}_{1} & =f_{1}\left(t, \mathbf{x}_{1}\right)+g(t, \mathbf{x}) \mathbf{x} \\
\dot{\mathbf{x}}_{2} & =f_{2}\left(t, \mathbf{x}_{2}\right)
\end{aligned}
$$

where $\mathbf{x}=\left[\begin{array}{ll}\mathbf{x}_{1}^{\top} & \mathbf{x}_{2}^{\top}\end{array}\right]^{\top}, \mathbf{x}_{1}:=\mathbf{e}_{\delta}^{e}, \mathbf{x}_{2}=\left[\mathbf{e}_{q \pm}^{\top}\left(\boldsymbol{\omega}_{d, w}^{b}\right)^{\top}\right]^{\top}$, $f_{1}\left(t, \mathbf{x}_{1}\right):=-\mathbf{S}\left(\boldsymbol{\omega}_{n, e}^{e}\right) \mathbf{e}_{\delta}^{e}-\mathbf{v}_{r}^{w}$,

$g(t, \mathbf{x}):=\left[\begin{array}{ll}\mathbf{0} & 2 \eta_{e, w} \mathbf{S}\left(\mathbf{v}_{r}^{w}\right)+2 \mathbf{S}\left(\boldsymbol{\epsilon}_{e, w}\right) \mathbf{S}\left(\mathbf{v}_{r}^{w}\right) \mathbf{0}\end{array}\right]$, and where $f_{2}\left(t, \mathbf{x}_{2}\right)$ represents the rotational dynamics in closed loop with a rotational controller. Consider first the unforced system $\dot{\mathbf{x}}_{1}=f_{1}\left(t, \mathbf{x}_{1}\right)$ written as

$$
\dot{\mathbf{x}}_{1}=-\mathbf{S}\left(\boldsymbol{\omega}_{n, e}^{e}\right) \mathbf{x}_{1}-\mathbf{v}_{r}^{w}
$$

Let a Lyapunov function candidate be defined as $V:=$ $\frac{1}{2} \mathbf{x}_{1}^{\top} \mathbf{x}_{1}$ which through differentiation and inserting (A.7) becomes $\dot{V}=-\mathbf{x}_{1}^{\top} \mathbf{v}_{r}^{w}$ since $\mathbf{x}_{1}^{\top} \mathbf{S}\left(\boldsymbol{\omega}_{n, e}^{e}\right) \mathbf{x}_{1}=0$. Note that both $\mathbf{x}_{1}=\left[\begin{array}{lll}\left\|\mathbf{e}^{n}\right\|_{\delta} & 0 & 0\end{array}\right]^{\top}$ and $\mathbf{v}_{r}^{w}=\left[\begin{array}{lll}V_{a} & 0 & 0\end{array}\right]^{\top}$ are positive with only components on the x-axis, such that $\dot{V}=-\left\|\mathbf{x}_{1}\right\| \beta_{v}$ where $\beta_{v}$ represents the lower bound of the airspeed. This means that as $\mathbf{x}_{1}(t) \rightarrow \mathbf{0}$, the trajectories will converge to the set $\mathcal{H}(\delta, \Delta)$. Hence, it follows by using Theorem 4.9 in [4] that the set $\mathcal{H}(\delta, \Delta)$ is uniformly asymptotically stable when $\mathbf{x}_{2}=\mathbf{0}$.

To prove that the cascade is uniformly asymptotically stable, three assumptions must be fulfilled in order to invoke the results on cascaded theory by [9]. Since the set $\mathcal{H}(\delta, \Delta)$ of the unforced system $\dot{\mathbf{x}}_{1}=f_{1}\left(t, \mathbf{x}_{1}\right)$ is uniformly asymptotically stable, it follows from converse theorems (e.g. [4]) that there exists a suitable $V\left(t, \mathbf{x}_{1}\right)$, and as such the first assumption in [9] is fulfilled. The second assumption requires that the interconnection term $g(t, \mathbf{x})$ has a linear growth bound. The interconnection term can be bounded as

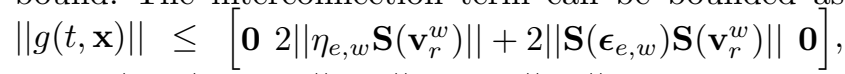
where $\left|\eta_{e, w}\right| \leq 1,\left\|\boldsymbol{\epsilon}_{e, w}\right\| \leq 1,\left\|\mathbf{v}_{r}^{w}\right\| \leq \beta_{V_{\max }}$, and consequently the second assumption is fulfilled. The third assumption states that the trajectories of system (A.6) must converge sufficiently fast to the origin through an integrability constraint. The sliding surface controller in [8] in closed loop with the rotational 
dynamics results in uniformly exponential stability of the equilibrium $\mathbf{x}_{2}=\mathbf{0}$. This means that $\left\|\mathbf{x}_{2}\right\| \rightarrow 0$ exponentially fast, thus fulfilling the third assumption. Hence, it follows by invoking Theorem 1 in [9] that the set $\mathcal{C}:=\left\{\mathbf{x}_{1} \in \mathbb{R}^{3}, \mathbf{x}_{2} \in \mathrm{S}_{e}^{3} \times \mathbb{R}^{3} \mid \delta \leq\left\|\mathbf{x}_{1}\right\| \leq \Delta, \mathbf{x}_{2}=\mathbf{0}\right\}$ of the cascaded system (A.5)-(A.6) is uniformly asymptotically stable.

\section{B Collision Avoidance}

The quaternion can be found by studying (11) resulting in (8). Equation (11) can be differentiated as

$$
\dot{\mathbf{c}}^{c}=-\mathbf{S}\left(\boldsymbol{\omega}_{e, c}^{c}\right) \mathbf{c}^{c}-\mathbf{R}_{e}^{c} \mathbf{S}\left(\boldsymbol{\omega}_{n, e}^{e}\right) \mathbf{R}_{n}^{e} \mathbf{c}^{n}+\mathbf{R}_{n}^{c} \dot{\mathbf{c}}^{n}
$$

where $\mathbf{S}\left(\boldsymbol{\omega}_{e, c}^{c}\right) \mathbf{c}^{c}=-\mathbf{S}\left(\mathbf{c}^{c}\right) \boldsymbol{\omega}_{e, c}^{c}$ with

$$
\mathbf{S}\left(\mathbf{c}^{c}\right)=\left[\begin{array}{ccc}
0 & 0 & \mp\left\|\mathbf{c}^{e}\right\| \\
0 & 0 & 0 \\
\pm\left\|\mathbf{c}^{e}\right\| & 0 & 0
\end{array}\right]
$$

which filters out any $\mathbf{y}^{c}$ components such that $\mathbf{S}^{\dagger}\left(\mathbf{c}^{c}\right) \dot{\mathbf{c}}^{c}=$ 0. Solving (B.1) for the angular velocity results in (9). With $\dot{\mathbf{p}}_{o}^{n}=\mathbf{0}$ equation (B.1) can be rewritten as

$$
\dot{\mathbf{c}}^{c}=-\mathbf{S}\left(\boldsymbol{\omega}_{e, c}^{c}\right) \mathbf{c}^{c}-\mathbf{R}_{e}^{c} \mathbf{S}\left(\boldsymbol{\omega}_{n, e}^{e}\right) \mathbf{R}_{n}^{e} \mathbf{c}^{n}-\mathbf{R}_{w}^{c} \mathbf{v}_{r}^{w}
$$

where $\dot{\mathbf{p}}^{n}=\mathbf{R}_{w}^{n} \mathbf{v}_{r}^{w}$ follows from Assumption 2 . Let the rotation matrix be written as $\mathbf{R}_{w}^{c}=\mathbf{I}+$ $2 \eta_{c, w} \mathbf{S}\left(\boldsymbol{\epsilon}_{c, w}\right)+2 \mathbf{S}^{2}\left(\boldsymbol{\epsilon}_{c, w}\right)$, which can be inserted into (B.3) as $\dot{\mathbf{c}}^{c}=-\mathbf{S}\left(\boldsymbol{\omega}_{e, c}^{c}\right) \mathbf{c}^{c}-\mathbf{R}_{e}^{c} \mathbf{S}\left(\boldsymbol{\omega}_{n, e}^{e}\right) \mathbf{R}_{n}^{e} \mathbf{c}^{n}-(\mathbf{I}+$ $\left.2 \eta_{c, w} \mathbf{S}\left(\boldsymbol{\epsilon}_{c, w}\right)+2 \mathbf{S}^{2}\left(\boldsymbol{\epsilon}_{c, w}\right)\right) \mathbf{v}_{r}^{w}$. Let the collision avoidance quaternion be the primary task, such that $\mathbf{q}_{n, d}=\mathbf{q}_{n, c}$, then by making $\left(\mathbf{e}_{q \pm}, \boldsymbol{\omega}_{d, w}^{b}\right) \rightarrow(\mathbf{0}, \mathbf{0})$ through rotational control, it follows that $\mathbf{q}_{c, w} \rightarrow\left[\begin{array}{llll}1 & 0 & 0 & 0\end{array}\right]^{\top}$. The system can now be written on cascaded form similarly as in (A.5)-(A.6), but where $\mathbf{x}_{1}:=\mathbf{c}^{c}$, $f_{1}\left(t, \mathbf{x}_{1}\right):=-\mathbf{S}\left(\boldsymbol{\omega}_{e, c}^{c}\right) \mathbf{c}^{c}-\mathbf{R}_{e}^{c} \mathbf{S}\left(\boldsymbol{\omega}_{n, e}^{e}\right) \mathbf{R}_{n}^{e} \mathbf{c}^{n}-\mathbf{v}_{r}^{w}$ and $g(t, \mathbf{x}):=\left[\begin{array}{ll}\mathbf{0} & 2 \eta_{c, w} \mathbf{S}\left(\mathbf{v}_{r}^{w}\right)+2 \mathbf{S}\left(\boldsymbol{\epsilon}_{c, w}\right) \mathbf{S}\left(\mathbf{v}_{r}^{w}\right) \mathbf{0}\end{array}\right]$. Consider the unforced system $\dot{\mathbf{x}}_{1}=f_{1}\left(t, \mathbf{x}_{1}\right)$ which can be written in original coordinates as $\dot{\mathbf{c}}^{c}=$ $-\mathbf{S}\left(\boldsymbol{\omega}_{e, c}^{c}\right) \mathbf{c}^{c}-\mathbf{R}_{e}^{c} \mathbf{S}\left(\boldsymbol{\omega}_{n, e}^{e}\right) \mathbf{R}_{n}^{e} \mathbf{c}^{n}-\mathbf{v}_{r}^{w}$. A Lyapunov function candidate can be defined as $V=\frac{1}{2}\left(\mathbf{c}^{c}\right)^{\top} \mathbf{c}^{c}$ which through differentiation results in $\dot{V}=0$. This means that as long as the collision avoidance quaternion is perfectly tracked, the relative distance between the obstacle and the UAV will neither increase nor decrease, such that the UAV will move in a circular trajectory around the obstacle. The rest of the proof follows as in Appendix A. Even though Theorem 1 in [9] considers the stability of cascades, similar arguments can be applied to prove collision avoidance. As $\mathbf{x}_{2} \rightarrow \mathbf{0}$ and $\dot{V} \rightarrow 0$, it follows that the distance to the obstacle goes to a constant, $\beta_{c}>0$. Hence, it follows that the position error between the obstacle and the UAV becomes lower bounded as $\left\|\mathbf{c}^{c}\right\| \geq \beta_{c}>0$, and collision is avoided.

\section{Proof of Theorem 1}

When the primary task is active, it follows from Lemma 3 that $\left\|\mathbf{h}^{c}\right\| \geq \beta_{h}>0 \forall t \geq t_{0}$. As the primary task is completed, it follows that $\mathbf{q}_{c, h}=\left[\begin{array}{llll}1 & 0 & 0 & 0\end{array}\right]^{\top}$ reducing (2) to $\mathbf{q}_{n, h}=\mathbf{q}_{n, c}=\mathbf{q}_{n, e} \otimes \mathbf{q}_{e, c}$ where the secondary task becomes fully active. Using Lemma 2 , the secondary task ensures that $\left\|\mathbf{c}^{c}\right\| \geq \beta_{c}>0 \forall t \geq t_{0}$. As the secondary task is completed, (2) is reduced to $\mathbf{q}_{n, h}=\mathbf{q}_{n, e}$ which by using Lemma 1 ensures that $\left\|\mathbf{e}^{e}\right\| \rightarrow \mathcal{H}(\delta, \Delta)$.

\section{References}

[1] G. Antonelli, F. Arrichiello, and S. Chiaverini. The null-space-based behavioral control for mobile robots. In Proceedings of IEEE International Symposium on Computational Intelligence in Robotics and Automation, Espoo, Finland, 2005.

[2] J. Borenstein and Y. Koren. The vector field histogram - fast obstacle avoidance for mobile robots. IEEE Transactions on Robotics and Automation, Vol. 7, No. 3:pp. 278-288, 1991.

[3] R. A. Brooks. A robust layered control system for a mobile robot. IEEE Journal of Robotics and Automation, Vol. RA2, No. 1:pp. 14-23, 1986.

[4] H. K. Khalil. Nonlinear systems. 3rd ed., Prentice Hall, ISBN 0-13-067389-7, 2002.

[5] O. Khatib. Real-time obstacle avoidance for manipulators and mobile robots. The International Journal of Robotics Research, Vol 5. No. 1:pp. 90-98, 1986.

[6] Y. Koren and J. Borenstein. Potential field methods and their inherent limitations for mobile robot navigation. In Proceedings of the IEEE Conference on Robotics and Automation, Sacramento, CA, USA, 1991.

[7] R. Kristiansen, A. Loría, A. Chaillet, and P. J. Nicklasson. Spacecraft relative rotation tracking without angular velocity measurements. Automatica, Vol. 45, No. 3:pp. 750-756, 2009.

[8] E. Oland, R. Schlanbusch, and R. Kristiansen. Underactuated waypoint tracking of a fixed-wing UAV. In Proceedings of the 2nd RED-UAS, Compiegne, France, 2013.

[9] E. Panteley and A. Loría. On global uniform asymptotic stability of nonlinear time-varying systems in cascade. Systems \& Control Letters, Vol. 33, No. 2:pp. 131-138, 1998.

[10] E. Rimon and D. E. Koditschek. Exact robot navigation using artificial potential functions. Transactions on Robotics and Automation, Vol. 8, No. 5:pp. 501 - 518, 1992.

[11] G. Roussos, D. V. Diamarogonas, and K. J. Kyriakopoulos. 3D navigation and collision avoidance for nonholonomic aircraft-like vehicles. Intl. Journal of Adaptive Control and Signal Processing, Vol 24, No. 10:pp. 900-920, 2010.

[12] B. L. Stevens and F. L. Lewis. Aircraft control and simulation. 2nd ed., Wiley, ISBN 978-0-471-37145-8, 2003.

[13] H. G. Tanner, S. G. Loizou, and K. J. Kyriakopoulos. Nonholonomic navigation and control of cooperating mobile manipulators. IEEE Transactions on Robotics and Automation, Vol. 19. No. 1:pp. 53-64, 2003. 